\title{
Por qué fracasa la deliberación y cómo podemos remediarlo. Una alternativa ética al enfoque neurocientífico
}

\author{
Why deliberation fails and how we can remedy it. An ethical \\ alternative to neuroscientific approach
}

PEDRO JESÚS PÉREZ ZAFRILLA*

\begin{abstract}
Resumen: Para la democracia deliberativa el acuerdo racional es el fin de los procesos deliberativos. Sin embargo, estudios actuales muestran que en ciertas ocasiones la deliberación, lejos de producir el acuerdo, genera procesos de polarización. Una aproximación a este problema de la polarización ha sido desarrollada desde la neurociencia por autores como Lakoff, Haidt, Hugo Mercier o Hélène Landemore. Así, en este artículo pretendemos, en primer lugar, abordar el enfoque de estos autores sobre la polarización. Esto permitirá extraer los errores que pueda haber en sus planteamientos. Finalmente, desarrollaremos una propuesta para corregir la polarización de los procesos deliberativos.

Palabras clave: Deliberación - desacuerdo polarización - neuropolítica - emociones.
\end{abstract}

\begin{abstract}
According to Deliberation rational agreement is the aim of deliberative processes. However, current studies show that often deliberation doesn't produce agreement, but polarization processes. An approach to the problem of polarization has been addressed from neuroscience by authors like Lakoff, Haidt, Hugo Mercier o Hélène Landemore. So, in this article we firstly aim to address the approach of these authors on polarization. This will enable to draw the errors this approach could have. Finally, we will expose an alternative proposal to correct polarization in deliberative processes.
\end{abstract}

Keywords: Deliberative democracy - disagreement - polarization - neuropolitics - emotions.

\section{Introducción}

La democracia deliberativa surgió allá por los años ochenta con el propósito de reivindicar el diálogo, el acuerdo y la transformación de preferencias como ejes de la participación política. Argumentan sus defensores, como Estlund o Bohman, que el diálogo, a través de la

Fecha de recepción: 20/05/2015. Fecha de aceptación: 06/01/2016.

* Profesor del Departamento de Filosofía (Universitat de València). E-mail: p.jesus.perez@uv.es. Líneas de investigación: filosofía política, teorías de la democracia, democracia deliberativa, neuroética, neuropolítica, psicología moral. Entre sus últimas publicaciones cabe destacar: «Is Deliberative Democracy an adaptive political theory? A critical analysis of Hugo Mercier's Argumentative Theory of Reasoning», Análise Social, vol.LI, núm.220 (3), 2016, pp. 544-564.

Este estudio se inserta en el Proyecto de Investigación Científica y Desarrollo Tecnológico FFI2016-76753-C2-1-P, financiado por el Ministerio de Economía y Competitividad. 
argumentación racional, el intercambio de información y la justificación pública, conducirá a una decisión epistémicamente superior que una tomada por la mera agregación de intereses (Freeman, 2000, 402; Bohman, 1996, 187). El carácter epistémico es precisamente, en nuestra opinión, el elemento definitorio de esta teoría de la democracia y no tanto el que se recurra a la deliberación como proceso de toma de decisiones (Pérez Zafrilla, 2009).

Sin embargo, la propuesta deliberativa parece chocar de frente con la realidad, y ello por dos razones. Por un lado, porque los ensayos prácticos de democracia deliberativa realizados desde la teoría política no han permitido concluir que la deliberación como método de toma de decisiones conduzca a la convergencia de actitudes y opinión. Más bien los resultados obtenidos resultan contradictorios (Mercier y Landemore, 2012, 243). Por otro, porque diversos estudios demuestran que los procesos deliberativos están sometidos a varias formas de polarización (Auber, Crott y Werner, 1992; Isenberg, 1986; Sunstein, 2002; Darley y Rusbult, 2001; Lord, Ross y Lepper, 1979). Así por ejemplo, a diario observamos cómo la deliberación sobre ciertos temas morales y políticos especialmente polémicos (como la política penitenciaria o el aborto), en lugar de conducir al acuerdo en torno a la posición apoyada en los mejores argumentos, se convierte en un choque de argumentos entre posiciones antagónicas. En estos casos, el intercambio de argumentos y evidencias, lejos de producir el acuerdo razonado, en muchas ocasiones conduce a los sujetos a extremos irreconciliables. Acabado el debate, cada uno se siente más reafirmado en sus posiciones iniciales y considera que su oponente o está sumido en el error o es un sujeto sectario que oculta unos intereses inconfesables bajo su defensa recalcitrante de posiciones que el primero considera inmorales (Schulz, 2010, 133).

De este modo, parece alumbrarse una grave disonancia entre, por un lado, la defensa que la democracia deliberativa hace del diálogo como el proceso dirigido al acuerdo racional entre personas con actitud razonable, y, por otro, el hecho evidente de que la deliberación pública parece conducir en ocasiones al enconamiento de posturas y el recelo entre los participantes. ¿A qué se debe, entonces, esta aparente contradicción entre el nivel normativo y el nivel de aplicación de la teoría deliberativa? Pero sobre todo, ¿dónde radica la causa de esa polarización y cómo puede remediarse?

Una respuesta a estas preguntas ha sido proporcionada recientemente desde la neurociencia. En concreto lo han hecho los autores centrados en el estudio de la deliberación, como George Lakoff, Jonathan Haidt, Hélène Landemore o Hugo Mercier. Por ese motivo, en este trabajo deseamos someter a estudio las propuestas realizadas por estos autores sobre las disonancias que presentan los procesos deliberativos y que derivan en formas de polarización. Esto permitirá, en segundo lugar, analizar las implicaciones que este fenómeno posee sobre la propuesta de la democracia deliberativa.

\section{La polarización desde la neurociencia}

\subsection{El enfoque emotivista}

La polarización en los procesos deliberativos ha sido abordada en la neurociencia, principalmente en el marco los estudios realizados desde la neuropolítica y el neuromárketing electoral. En esta perspectiva, autores como Lakoff y Haidt han mantenido un enfoque 
emotivista. Afirman estos autores que los juicios morales no son fruto del razonamiento sosegado, como se mantiene tradicionalmente desde la filosofía y desde la psicología cognitiva de Kohlberg, sino que tienen un origen inconsciente en la activación de nuestras sinapsis cerebrales. Los juicios morales serían el reflejo en nuestra consciencia de una valencia emocional de aprobación o rechazo de un estímulo en función de su incidencia sobre nuestro bienestar. Así también, el razonamiento sería en la mayoría de las ocasiones un proceso motivado, y por ende, sesgado, guiado por los deseos y actitudes de tal forma que las conclusiones de aquél serán acordes con éstos (Kunda, 1990, 482). ${ }^{1}$ La reflexión representa así un proceso post hoc dirigido a crear argumentos que confirmen la impresión inicial adoptada de modo intuitivo.

Esto hace que para Lakoff y Haidt constituya un error defender que la deliberación se asienta en un intercambio de argumentos dirigidos a tomar la mejor decisión (Lakoff, $2009,8)$. El origen intuitivo de los juicios morales y el carácter motivado que posee el razonamiento convierten en una ilusión la expectativa que poseen los sujetos de que sus argumentos convenzan a su interlocutor moralmente motivado (Haidt, 2001, 823). La evaluación sesgada que cada cual realiza de los argumentos del oponente, así como la actitud contraria que ambos poseen sobre el asunto, impiden que el intercambio de evidencias y argumentos presentados por cada cual conduzca al acuerdo racional entre las partes. Porque el posicionamiento de los participantes no se asienta en evidencias sino en intuiciones. De esta forma, la deliberación quedará reducida a un choque de razones post hoc que apoyan la tesis hacia la que cada uno posee una actitud positiva. Así, donde los sujetos creían encontrar una pugna entre diferentes criterios de justicia con el objetivo de tomar la mejor decisión mediante el reconocimiento del mejor argumento, en realidad existe una oposición de actitudes sobre el asunto.

Por ese motivo, para estos autores no tiene sentido centrar la deliberación en la refutación de los argumentos del oponente. Este es un proceso fútil que conduce inevitablemente a la polarización de posturas en el diálogo (Haidt, 2012, 48). El hecho de que los argumentos presentados no sean capaces de convencer al interlocutor genera en los participantes unos sentimientos de frustración y desconfianza hacia el interlocutor. Una consecuencia de esos sentimientos será la polarización del diálogo. Cada cual piensa que si la presentación de evidencias y argumentos adecuados no logra convencer a su interlocutor es, o porque éste es estúpido y no llega a comprender la realidad que al hablante le parece evidente, o porque es malo, y entonces no se quiere dejar convencer porque mantiene unos intereses espurios; esto es, cada uno pensará que su interlocutor no está motivado moralmente.

Este choque entre percepciones inconmensurables sobre el objeto del debate hace, según Haidt, que la deliberación derive en un proceso de polarización en el que la posición del interlocutor no es percibida como algo que puede contribuir al logro del bien común, sino como una posición errónea que debe ser refutada. Cada cual trata de elaborar mejores argumentos para defender su postura. Pero como esos argumentos no logran convencer al interlocutor, el resultado es un mayor enconamiento de posiciones que imposibilita el acuerdo.

Desaparece así la idea desarrollada desde la democracia deliberativa según la cual la deliberación constituye un proceso argumentativo mediante el que todos los participantes llegan

1 Este es el conocido sesgo del razonamiento motivado. 
a un punto de vista compartido (Cohen, 1986). En su lugar, Lakoff y Haidt entenderán que el modo más eficaz de cambiar el posicionamiento del interlocutor será cambiar su actitud mediante la persuasión emocional. Para ello los recursos más eficaces serán las metáforas y otros elementos retóricos, debido a su capacidad para activar las fuentes inconscientes de la cognición humana. De acuerdo a estos autores, los contenidos mentales no son neutrales ni son tampoco compartidos por todos los sujetos. Los contenidos de nuestra mente sólo cobran sentido dentro de unas narrativas o marcos. Los marcos son las estructuras mentales con las que nos aproximamos a la realidad y le dan sentido a ésta. Los marcos están fijados en las redes neuronales de asociación que se activan en nuestro cerebro y que conectan de forma inconsciente las metáforas con emociones y pensamientos concretos dentro de un marco mental. Así cada concepto cobra sentido dentro de un marco, y ese sentido queda unido a una reacción automática de aprobación o rechazo (Lakoff, 2009, 93; Westen, 2007, 103; García-Marzá, 2012, 93).²

En el ámbito deliberativo, la significación metafórica de los conceptos produce de manera no reflexiva la aceptación o rechazo de los argumentos. Ello dependerá de si los conceptos empleados producen la activación del marco adecuado en el interlocutor. De esta forma, al acuerdo se llega logrando que el oyente adopte nuestro marco mediante el recurso a la retórica. En palabras de Haidt: «cada argumento es en realidad un intento de enmarcar el asunto con el fin de presionar un botón emocional, provocando diversos flashes de intuiciones en el oyente» (Haidt y Bjorklund, 2007, 192), y enmarcar no es otra cosa que activar ciertas redes neuronales que producen un determinado marco, el que interesa al hablante (Castells, 2009, 197) Por el contrario, presentar datos objetivos y argumentos racionales incapaces de activar un marco mental no producirá el acuerdo en el diálogo. Porque el oyente no concede valor a ningún dato ni argumento que caiga fuera de su marco mental. Esta es la clave para entender por qué el esperar a que el oyente analice los argumentos de forma objetiva y cambie de opinión sólo genera frustración en el hablante. Según Haidt, esa es una expectativa inútil pues sólo es posible cambiar la actitud del oyente empleando la metáfora apropiada para cambiar su marco mental, no recurriendo a evidencias.

\subsection{La Teoría Argumentativa del Razonamiento}

Otra propuesta que desde la neurociencia ha analizado el problema de la polarización en los procesos de deliberación es la denominada Teoría Argumentativa del Razonamiento (en adelante TAR), desarrollada por autores como Hugo Mercier, Hélène Landemore y Dan Sperber. Esta teoría, de la mano de Mercier y Landemore principalmente, analiza los procesos deliberativos desde la psicología evolutiva. Pero frente a otros autores como Lakoff y Haidt, la TAR se caracteriza por su pretensión de reivindicar desde la neurociencia las tesis defendidas desde la democracia deliberativa. Por ese motivo, resulta de especial interés contraponer el enfoque de Mercier y Landemore al de Haidt y Lakoff.

La tesis principal de la TAR es que la deliberación es una forma de razonamiento llevada a cabo dentro de unas condiciones concretas que conducen a los participantes a tomar un acuerdo racional en torno a la mejor decisión. En cambio, cuando esas condiciones no se

2 Esta reacción automática es lo que Haidt denomina intuiciones. 
cumplen, no nos encontraríamos propiamente ante una forma de deliberación sino ante otras formas de razonamiento, como la discusión o la tertulia (Landemore y Mercier, 2012, 919). Estos últimos procesos, a diferencia de la deliberación, están viciados por sesgos cognitivos que provocan la polarización de posturas. Esta es sin duda una tesis muy atrevida. Con ella Mercier y Landemore no sólo están defendiendo un modelo de deliberación similar al defendido por los autores de la democracia deliberativa; incluso dicen que la deliberación, por su propia lógica, no produce polarización. Sólo hay polarización en procesos de razonamiento que propiamente no son deliberativos. Para comprender el planteamiento de Mercier y Landemore deberemos proceder analizando los dos elementos que dan cuerpo a la deliberación en la TAR: el razonamiento y el contexto social. Comencemos aludiendo al razonamiento.

Frente a la concepción mayoritaria dentro de la neurociencia, Mercier (2012, 260) rechaza que el papel del razonamiento sea la creación de juicios mediante la reflexión privada. Por el contrario, defenderá que la facultad propia del razonamiento es la argumentativa, dirigida a facilitar la comunicación entre las personas (Mercier y Sperber, 2011, 60). Según la TAR la función del razonamiento se desarrolló evolutivamente por sus ventajas adaptativas en las relaciones intersubjetivas. El intercambio de información en el proceso argumentativo proporcionará múltiples ventajas. Entre ellas podemos citar: mantener la reputación individual, la resolución de problemas de cualquier índole, la detección de free riders o la toma de mejores decisiones, por ejemplo. Con ello, el papel del razonamiento dentro de los procesos comunicativos será el de contribuir a la efectividad de esa comunicación mediante la discriminación entre la información fiable de la que no lo es.

El razonamiento se compone de dos procesos cognitivos. Landemore y Mercier definen el razonamiento como «un mecanismo cognitivo específico que trata de encontrar y evaluar razones, de tal forma que los individuos puedan convencer a otras personas y evaluar sus argumentos» (Landemore y Mercier, 2012, 914; Mercier y Sperber, 2011, 59). Tales procesos están bien diferenciados y desempeñan funciones diferentes. En primer lugar está el ejercicio de atender y evaluar las razones que hay a favor y en contra de un asunto en el marco de un proceso deliberativo. En la evaluación el sujeto pretende analizar los argumentos del interlocutor para discriminar la fiabilidad de éste así como de la información que suministra y determinar si debe aceptar o no su contenido. Esta discriminación de la información se llevará a cabo mediante el mecanismo cognitivo de la vigilancia epistémica, desarrollado evolutivamente, según los autores de la TAR, para permitir la supervivencia de los sujetos en los contextos comunicativos (Sperber, Clément, Heintz, Mercier, Origgi y Wilson, 2010). Esa evaluación de los argumentos del interlocutor permite al sujeto, llegado el caso, reconocer errores en su percepción de la realidad (Mercier, 2011, 143; Landemore y Mercier, 2012, 915). Cuando ello sucede, el sujeto reconsiderará su propia posición y la cambiará por otra.

Por otro lado está la función de buscar y articular argumentos. Esta función posee un doble objetivo. El primero es responder a los argumentos del oponente cuando no se consideran concluyentes; el segundo es tratar de convencer al interlocutor de nuestra posición. Este doble proceso de evaluación y argumentación permite a los sujetos tomar mejores decisiones en el marco de procesos comunicativos (Mercier, 2011, 132).

De esta forma, la TAR coincide con autores como Joshua Greene en reivindicar la facultad del razonamiento en los procesos intersubjetivos, frente al planteamiento de Haidt, para quien la deliberación se reduce a un proceso de persuasión emocional (Paxton y 
Greene, 2010; Haidt, 2001, 823). Pero, frente a Greene, la TAR comparte con Haidt que la reflexión, salvo excepciones, sólo es fructífera cuando se realiza a partir de la información proporcionada por terceras personas. ${ }^{3}$ Así pues, el ámbito propio del razonamiento es el de las relaciones comunicativas y no la reflexión privada. Por ello, el segundo elemento que caracteriza a la TAR, y que paso a analizar, es el contexto social.

Para que el razonamiento pueda cumplir adecuadamente la función de conducir a las partes al acuerdo racional, debe desarrollarse en unas condiciones adecuadas, lo que Mercier y Landemore denominan «condiciones normales». Serán precisamente esas condiciones apropiadas del uso del razonamiento también las que caractericen la deliberación. En concreto, esas condiciones son fundamentalmente tres: La primera es que haya una confrontación entre puntos de vista discrepantes sobre el tema abordado (Mercier y Landemore, 2012, 248; Thompson, 2008). La segunda es que se evalúen los argumentos del interlocutor (Mercier, 2011, 140; Landemore y Mercier, 2012, 916). Por último, los sujetos deben estar motivados adecuadamente (Mercier y Sperber, 2011, 61). Es decir, estarán orientados a la verdad y a no tratarán de defender en el diálogo una posición de forma recalcitrante. En estas condiciones el intercambio de argumentos permitirá tomar la mejor decisión mediante la evaluación de la información.

Según Landemore y Mercier estas condiciones no se cumplen en ciertos contextos comunicativos, como la comunicación dentro de grupos afines descrita por autores como Sunstein (2002). Esta forma de comunicación no constituye una forma de deliberación porque no cumple con las condiciones señaladas por Mercier y Landemore: no hay divergencia de opiniones (porque el conjunto de argumentos presentes están todos dirigidos en una misma opinión compartida por todos); y, lo que es más importante, tampoco se produce una evaluación de los argumentos del interlocutor, sino meramente una producción de argumentos, cada vez más radicales, lo que propicia la polarización del grupo (Mercier y Landemore, 2012, 247).

Pero el criterio definitorio de la deliberación no es propiamente que haya una diversidad de posiciones en el diálogo, sino que esas posiciones estén realmente contrapuestas y los argumentos presentados estén dirigidos a rebatir las tesis del interlocutor. Porque la mera heterogeneidad de planteamientos no implica que exista una contraposición entre ellos. El elemento clave de la deliberación para Landemore y Mercier es el proceso de la evaluación. No en vano, lo que define la deliberación, como ya dijera Aristóteles (2000), es el discernimiento de las razones que hay a favor y en contra de un asunto (Manin, 2005, 15). De ahí que Mercier y Landemore distingan entre los procesos de argumentación y de evaluación en el razonamiento. El primero es un proceso sesgado que sólo articula razones favorables a las tesis que defiende el sujeto. Por su parte, la evaluación trata de discernir la información valiosa que pueda haber en las razones aportadas por el interlocutor para tomar una buena decisión. Esto es, la evaluación conlleva una disposición a dejarse convencer por el mejor argumento.

De esta forma, el diálogo en grupos de sujetos afines, o en ámbitos de pluralidad de planteamientos pero en los que no se lleva a cabo esa evaluación del planteamiento del

3 Sendas teorías, la TAR y el intuicionismo social, aceptan la posibilidad de que exista una deliberación interna que lleve a la producción de juicios no sesgados. Pero ello requerirá de un esfuerzo cognitivo especial que no se produce de forma habitual. 
interlocutor, deben ser denominados mejor como tertulias o discusiones pero no como deliberación (Landemore y Mercier, 2012, 927; Mercier y Landemore, 2012, 246). En esos otros procesos de comunicación impera el sesgo de confirmación, que conduce a la elaboración de razones dirigidas a la reafirmación en la opinión inicial (Lord, Ross y Lepper, 1979, 2099; Kunda, 1990, 484). Porque en ellos el sujeto no trata de llegar al acuerdo racional, sino defender de forma recalcitrante una tesis sin contemplar que pueda estar equivocado. Esta actitud irrazonable que poseen los sujetos y la ausencia de una evaluación crítica de los argumentos del interlocutor son los elementos que dirigirán el razonamiento hacia la polarización de posturas en lugar del acuerdo.

En consecuencia, Mercier y Landemore parecen coincidir con Lakoff y Haidt en que la causa de la polarización está en una concepción errónea de la deliberación. Para Lakoff y Haidt la polarización es fruto de la expectativa frustrada de lograr el convencimiento racional del interlocutor. Sin embargo, un enfoque correcto del proceso deliberativo, entienden, llevaría a asumir que la deliberación es en realidad un mecanismo de persuasión emocional dirigido a imponer mediante metáforas nuestro marco sobre el marco del interlocutor. Por ese motivo, en ese proceso de persuasión no hay lugar para el convencimiento racional ni la polarización de posturas. Por su parte, Landemore y Mercier entienden que en la deliberación no se produce polarización de posturas (Pérez Zafrilla, 2016). Pero, a diferencia de Lakoff y Haidt, para Mercier y Landemore en la deliberación es posible el acuerdo racional, gracias al desarrollo de sendos procesos cognitivos de argumentación y evaluación que componen el razonamiento y que tendrían un origen evolutivo.

\section{Las emociones morales en la deliberación}

De esta forma, los autores analizados defienden que propiamente la polarización no forma parte de los procesos deliberativos. Para Lakoff y Haidt esto es así porque los procesos de polarización son fruto de la una ilusión de la psicología cognitivista; para Mercier y Landemore, por su parte, la polarización es fruto de tipos de razonamientos no deliberativos, carentes de un intercambio crítico de argumentos. Sin embargo, como trataremos de mostrar a continuación, ambos planteamientos son erróneos, ya que no logran reconocer la dimensión moral que poseen las emociones reactivas en el proceso deliberativo. Frente a lo defendido por Lakoff y Haidt, las emociones reactivas poseen un carácter racional; frente a Landemore y Mercier, esas emociones son el elemento clave para comprender que los procesos de polarización ocupan un lugar dentro de los procesos deliberativos.

Hemos de empezar por señalar que ambos planteamientos poseen concepciones erróneas de la deliberación. La deliberación ni es una forma de persuasión emocional ni tampoco un mecanismo de razonamiento de carácter adaptativo dirigido a mantener la supervivencia de los participantes en los contextos comunicativos. La deliberación se rige por unos parámetros epistémicos y morales ausentes en las propuestas de estos autores. Así, frente a lo defendido por el intuicionismo social de Haidt, los juicios morales no son una expresión subjetiva de agrado o desagrado hacia algo. Los juicios morales poseen una pretensión de universalidad. Esto significa que la alternativa a lo afirmado como justo no se considera aceptable (Cortina, 2007, 18). Del mismo modo, el juicio moral entraña una pretensión de intersubjetividad: se espera que ese juicio sea aceptado racionalmente por todos. 
Esta es una idea clave que articula el proceso deliberativo, como nos recuerdan también desde la democracia deliberativa. Para los autores de esta teoría política, al argumentar los sujetos presuponen un criterio de justicia dirigido al bien común (Cohen, 1986, 26-38; Christiano, 1996, 29-30). Ese criterio se presupone intersubjetivo, de tal forma que al argumentar los sujetos albergan implícitamente una expectativa de alcanzar la comprensión y el acuerdo entre todos. Esto hace además, que quien defienda algo en un proceso deliberativo lo haga porque lo considera justo, o al menos mejor que las alternativas presentes, no porque satisfaga sus intereses egoístas. La pretensión de intersubjetividad y, en consecuencia, del convencimiento racional, son así elementos constitutivos del proceso deliberativo. De esta forma, la deliberación se asienta sobre una expectativa recíproca de convencimiento racional. Cada sujeto expone sus argumentos con la pretensión de convencer racionalmente al resto y a la vez acepta ser convencido por otro argumento mejor que le haga ver a todos que esa otra propuesta es la correcta. Así, la deliberación queda convertida en un ajustamiento de creencias entre los ciudadanos sobre lo que constituye la mejor opción a tomar, a la luz del reconocimiento del mejor argumento (Cohen, 1986, 34).

Pero también la deliberación se asienta sobre unos principios morales básicos. Al deliberar con otra persona, los sujetos presuponen el deber de reconocer al otro como interlocutor válido (Cortina, 2007, 181). Esto es así ya que en el diálogo los sujetos reconocen una relación moral. Dicha relación pasa por el deber de reconocer que la relación con el otro en el diálogo debe asentarse sobre la simetría y el recurso a razones que el otro pueda razonablemente aceptar, quedando excluida toda forma de manipulación. Por otro lado, quien participa en un debate reconoce el deber de estar guiado por intereses universalizables, así como por un criterio de justicia pretendidamente intersubjetivo. Quien no respetara este deber y se guiara por intereses egoístas propiamente no estaría deliberando sino negociando, faltando por tanto a esa expectativa recíproca de convencimiento que articula la deliberación.

Estas condiciones, ausentes en los planteamientos de Lakoff, Haidt y Landemore y Mercier, ponen en cuestión las tesis de estos autores sobre el fenómeno de la polarización. Como hemos señalado, los sujetos presuponen que quienes escuchen sus razones serán convencidos y apoyarán la propuesta realizada. En consecuencia, cuando el acuerdo no es posible, por las diferencias cognitivas que separan a las personas, y el interlocutor A contraargumenta desde una posición que B no comparte, B podrá pensar que A no está moralmente motivado en la deliberación al no aceptar el argumento presentado antes por B y que éste considera el mejor. Estos juicios sobre la falta de motivación moral del interlocutor suelen producirse en los procesos deliberativos cuando se abordan asuntos especialmente polémicos, en los que el proceso de evaluación crítica no conduce a la convergencia de posiciones. Pero esos juicios no se tratan de una ilusión, como cree Haidt, ni de un sesgo cognitivo, como defienden Mercier y Landemore. Al contrario, representan un elemento integrante y plenamente racional de la deliberación. Para mostrarlo creo conveniente aludir a los sentimientos reactivos que suelen acompañar a dichos pensamientos.

Los sentimientos de recelo que aparecen cuando el interlocutor no es convencido por nuestros argumentos, poseen un claro carácter moral. Son estas unas emociones morales, como la ira, el resentimiento o la indignación. Las emociones morales se caracterizan por su contenido cognitivo. Permiten al sujeto percibir situaciones donde se está violando una norma moral. Esto es posible porque, como señala Cortina $(2007,85)$, esas emociones pre- 
suponen la existencia entre los sujetos de una relación regida por unos principios morales que debe ser respetada. Pero, y esto es lo más importante, esa relación moral se fundamenta en una expectativa recíproca impersonal de cumplimiento, dirigida tanto a los demás como hacia uno mismo. Sentimos culpa cuando reconocemos que no nos hemos comportado de un modo que es exigible a todos en virtud de esa expectativa recíproca, e indignación cuando sentimos que ese comportamiento inmoral lo ha hecho otro (Cortina, 2011a, 144; Habermas, 1965, 66). Algo parecido sucede en la deliberación. Los sentimientos reactivos de frustración, recelo o indignación presentes en el debate, así como la consecuente creencia de que tal vez el interlocutor no esté motivado moralmente, reflejan que la deliberación pública se asienta sobre unos presupuestos morales de los que los sujetos son plenamente conscientes. Porque, como vimos, la expectativa recíproca de motivación moral es una condición inherente a la argumentación. Las personas sienten indignación o recelo al comprobar que su interlocutor no se convence por los argumentos presentados y piensan que éste no está motivado porque la relación dialógica se asienta sobre unos parámetros morales que deben ser respetados.

Por ejemplo, nosotros distinguimos entre las formas aceptables y no aceptables de comunicación en el ámbito deliberativo. En la deliberación se admite la argumentación racional pero no la demagogia o las falacias, al representar una forma de manipulación que busca imponer intereses egoístas. Por ese motivo los sentimientos reactivos surgen en la deliberación cuando alguien emplea esos recursos. Los sentimientos reactivos nos advierten de que alguien está violando los compromisos éticos que deben ser respetados en el proceso dialógico. Esos sentimientos reactivos nos revelan de forma inmediata esa carencia de actitud ética en el interlocutor, por ejemplo, al intentar manipular nuestros sentimientos o al intentar engañarnos. Dicho de otro modo, estos sentimientos reactivos representan la dimensión cordial de la razón por la que se reconoce una relación moral que no está siendo respetada (Cortina, 2007, 193).

Por otro lado, esos sentimientos reactivos surgen al comprobar que el interlocutor no es convencido por nuestros argumentos, produciéndose entonces los conocidos procesos de polarización. Este es un caso más complejo. Para analizarlo debemos partir de dos elementos interrelacionados. El primero son los dos aspectos de la fenomenología moral que los autores antes analizados no tienen en cuenta y a los que ya hemos hecho referencia: las pretensiones de universalidad e intersubjetividad que acompañan a los juicios morales. El segundo elemento es el hecho de que los sujetos deliberan desde marcos cognitivos inconmensurables (MacIntyre, 1987; Gaus, 1997).

La tesis de la pluralidad de marcos cognitivos que, como hemos visto, está presente entre neurocientíficos, ha sido defendida en el ámbito filosófico desde tiempo atrás. De hecho, el rechazo del modelo de razón universalmente compartida es un elemento característico de corrientes filosóficas como la hermenéutica o la tradición comunitarista. Autores como Gadamer, MacIntyre, Sandel o Taylor, pero también desde la democracia deliberativa, como Gutmann y Thompson o Gerald Gaus, han señalado (aunque desde diferentes planteamientos) que nuestro pensamiento se articula en torno a marcos proporcionados por la comunidad a la que pertenecemos. En ellos los distintos términos adquieren su sentido. Incluso para algunos de estos autores es en esos marcos en los que las personas forman su identidad.

Así, por ejemplo, señala Taylor que la valoración moral está dentro de un "marco referencial" que da sentido a nuestras vidas. Pero, frente a las tesis de la sociobiología, ese 
marco moral no se asienta en respuestas intuitivas a los estímulos recibidos del exterior, sino que está constituido por unos bienes a los que reconocemos una dignidad y valor superior independientes de nuestros deseos o inclinaciones. Los marcos proporcionan a las personas tanto lo que reconocen como valioso como el sentido mismo que adquiere lo valioso para cada persona (Taylor, 1996, 34). Nuestro marco moral viene dado, según Taylor $(1996,46)$, por aquellos colectivos con los que estamos fuertemente comprometidos y que forjan también nuestra identidad. Es desde su pertenencia a tales grupos como el sujeto se alfabetiza moralmente. No obstante, en nuestras sociedades modernas conviven grupos diversos con los que se identifican los sujetos. En ellos se reconocen bienes diferentes que pueden llegar a ser opuestos. De esta forma, los marcos que articulan el pensamiento moral de las personas de diferentes grupos son distintos y estarán enfrentados en su ontología moral.

Estas diferencias en principio no harían imposible el acuerdo político, ya que, frente a lo defendido por los liberales, el pluralismo epistémico no representa en sí mismo un obstáculo para el entendimiento entre personas razonables (Smith, 1997, 1.611). Sin embargo, en el tratamiento de ciertos asuntos morales y políticos el acuerdo se hace imposible, produciéndose además procesos de polarización. Esto sucede en aquellos casos en que entran en juego valores que los sujetos consideran fundamentales y, por tanto, inviolables. Entonces la opción contraria a la que defienden la consideran intolerable (Talisse, 2009, 18). En estas situaciones los planteamientos que hay enfrentados sobre el asunto se asientan en marcos referenciales que resultan inconmensurables y entre los que el acuerdo no es posible. Los marcos referenciales proporcionan a los sujetos ontologías morales diferentes con las que comprender la realidad. ${ }^{4}$ Así, en estos casos ciertos conceptos que articulan el debate adquieren una dimensión metafísica o moral (dependiendo del caso) que afecta a la valoración que los sujetos hacen de las cuestiones de hecho (Gutmann y Thompson, 1997, 73). Es así como los conceptos articuladores del caso adquieren caracterizaciones diferentes para cada una de las partes, como sucede con el concepto de «vida digna» en el caso de la eutanasia o el aborto, o el de «calidad de la educación» en los debates sobre educación. Lo mismo sucede con los valores morales o con las virtudes, que adquirirán caracterizaciones distintas para los individuos. Esto se debe a que los marcos referenciales se nutren de las diferentes tradiciones que dan un sentido particular a esos valores y a las virtudes (Taylor, 1996, 36; MacIntyre, 1987, 227-232).

Estas diferencias cognitivas explican la aparición de las emociones reactivas en las ocasiones en que chocan valores que los sujetos consideran fundamentales. Como señala Habermas, los sujetos al argumentar presuponen que su interlocutor asigna a los términos el mismo sentido que ellos les otorgan (Habermas, 1998, 66). Sin embargo, lo que no advierten es que el sentido que ellos asignan al concepto es el propio de su marco referencial. En cambio, en otro marco el mismo término puede adquirir una connotación distinta, que además resulta inconmensurable. Por ejemplo, a un pro-vida le irrita que un abortista apele al «derecho a abortar», por cuanto no puede haber derecho a quitar la vida a otro ser humano. En cambio, el abortista no entiende que el derecho a abortar implique matar, sino

4 Aquí entran en juego también las influencias sociales o familiares que rodean al sujeto y que le llevan a tener percepciones particulares de los hechos que pueden diferir incluso de sus doctrinas comprehensivas. En este sentido afirma Rawls que las personas no poseen doctrinas parcialmente comprehensivas y no plenamente comprehensivas. 
ejercer un acto de libertad con el propio cuerpo. Pero esto es justamente lo que no percibe el pro-vida desde su marco referencial, pues para él el aborto entraña un crimen. De ahí que la defensa del derecho al aborto genere irritación en el pro-vida. En sentido contrario, al proabortista le irrita que su defensa del aborto se equipare con la defensa del crimen, pues él no lo considera así.

En consecuencia, las razones construidas desde lo moralmente significativo para uno pueden no ser significativas para su interlocutor. De esta forma cada posición se hace impermeable a las razones del oponente, lo que hará irreconciliables las posiciones presentes en el diálogo. Así, al no resultar convincentes las razones del interlocutor, cada cual pensará que su interlocutor en realidad carece de buenas razones con que justificar su posición. Es más, a los sujetos les indigna que el oponente no reconozca el valor moral de sus posiciones y continúe defendiendo la tesis contraria, que aquél considera inmoral desde su perspectiva. En consecuencia, a cada participante le parecerá que su interlocutor no se quiere dejar convencer porque defiende intereses egoístas, llegándose así a la polarización de posiciones.

El resultado será una polarización de las posiciones hacia los extremos opuestos. El motivo es que los sujetos que ven afectadas sus convicciones fuertes sobre un asunto en la deliberación desde unas razones a las que no reconocen valor moral reaccionan de forma normal polarizando su posición. Este fenómeno no es, por tanto, un hecho irracional ni ajeno a la deliberación, sino, al contrario, una consecuencia normal del proceso deliberativo. El sujeto no puede mantener en la deliberación una disposición de apertura hacia una posición en la que no reconoce un valor moral sino, al contrario, la defensa de una alternativa que considera irrazonable. De ahí que la tendencia sea a reforzar su posición llevándola a un extremo en lugar de hacia la convergencia. En cambio, cuando se delibera sobre cuestiones en las que no se produce un choque entre convicciones profundas de los sujetos, la tendencia normal será la convergencia de posiciones (Wojcieszak, 2011, 609).

\section{Corregir la polarización}

La pregunta a la que finalmente hemos de responder es una que hacíamos al comienzo: cómo es posible corregir el proceso de polarización en el marco de la democracia deliberativa. Aun aceptando que la polarización sea un fenómeno comprensible dentro de la deliberación, eso no quiere decir que debamos resignarnos a él. Es posible corregir su aparición, y el remedio para ello está en la educación de las emociones reactivas.

Desde la democracia deliberativa se subraya la necesidad de que el ciudadano esté formado en unas virtudes que le permitan deliberar adecuadamente (Cortina, 2011b, 27-28). Algunos autores, como Richardson o Bohman, inciden en aquellas habilidades dirigidas a empoderar al individuo en el proceso deliberativo, como la capacidad de introducir en el debate temas que afectan al sujeto (Richardson, 2002, 90; Bohman, 1996, 132). Sin embargo, otros, como Gutmann y Thompson, ponen el acento en la necesidad de reforzar unas virtudes que hacen referencia a la actitud que el individuo debe tener hacia los interlocutores con los que está en franco desacuerdo. Entre estas virtudes cabe destacar la que estos autores denominan «magnanimidad cívica». Esta virtud consiste en la disposición a reconocer que el interlocutor es una persona bienintencionada y que no esconde tras sus planteamientos estrategias espurias ni intereses egoístas, aunque nuestros argumentos no le resulten convincentes (Gutmann y 
Thompson, 1997, 82-85). Dicho de otro modo, se debe reconocer al interlocutor como persona razonable. Así también, la magnanimidad cívica implica la disposición a reconocer el valor moral de las posiciones del interlocutor y que los argumentos de éste puedan transformar nuestro punto de vista. Finalmente, implica la disposición, por un lado, a reconocer que el desacuerdo es un hecho inevitable y, por otro, a minimizar el desacuerdo y a encontrar puntos de encuentro desde el reconocimiento de que sus posiciones son inconmensurables.

El fomento de la magnanimidad cívica permitirá a los individuos reconocerse mutuamente como personas razonables. Pero sobre todo les permitirá aceptar que el desacuerdo no es fruto de la carencia de motivación moral del interlocutor, sino del choque de esquemas cognitivos. De esta forma, se podrán cultivar en los sujetos actitudes que debiliten esos sentimientos reactivos que surgen actualmente ante el fracaso del convencimiento racional. Porque cambiando la forma de valorar el desacuerdo en el diálogo, se puede lograr también que nuestras emociones ante él sean distintas (Cortina, 2007, 86). Ahora bien, esto no significa que las emociones reactivas de indignación no sean nunca correctas. Lo son en aquellas circunstancias en las que se percibe una incoherencia entre lo defendido por el interlocutor y lo que hace realmente. Porque en esta situación de hipocresía la emoción moral nos revelaría la falta de actitud razonable del interlocutor al defender unos planteamientos que él realmente no cree.

Gracias al cultivo de la magnanimidad cívica, el desacuerdo ya no generará frustración ni recelo, sino respeto y aceptación. Así también, el fomento de la magnanimidad cívica será clave para lograr mejores acuerdos en el proceso deliberativo. El modo de hacerlo será fijando metas más realistas en el diálogo. Admitiendo el carácter inevitable del desacuerdo (reconocido incluso por la mayoría de autores de la democracia deliberativa) y la necesidad de tomar decisiones en un tiempo prudencial (pues el diálogo no puede alargarse indefinidamente), los sujetos ya no se fijarán como objetivo convencer de tesis fundamentales. Los objetivos de la deliberación serán distintos, por ejemplo medidas de alcance medio que no satisfagan del todo las metas de ninguna de las partes. Con ello se podrá aminorar el desacuerdo y al mismo tiempo dar respuesta a los problemas. Ahora bien, esto no significa en absoluto renunciar a la deliberación y apostar por un método de negociación para resolver los problemas. Ello es así porque la magnanimidad cívica presupone la actitud ética de los participantes, mientras que la negociación está guiada por la búsqueda de intereses egoístas y la renuncia a la argumentación racional en favor de la demagogia. Por ello precisamente, para lograr el acuerdo racional en contextos de desacuerdo profundo los sujetos deben renunciar a convencer a su interlocutor sobre aquellos aspectos en los que no cabe el acuerdo por la inconmensurabilidad de planteamientos.

Es así, finalmente, como se podrá evitar también la polarización en los procesos deliberativos. Cuando se parte del reconocimiento de la actitud bienintencionada del interlocutor y de la imposibilidad del acuerdo total sobre las convicciones que el sujeto tiene, nadie polarizará su postura. Más bien la tendencia natural será hacia la convergencia de posiciones en torno a unas medidas más limitadas. Incluso cuando se percibe la hipocresía en la posición defendida por el interlocutor tampoco se producirá la polarización. En esas ocasiones lo más prudente será sin duda dar por terminado el diálogo. Porque, como dijimos anteriormente, quien no mantiene una actitud ética en el diálogo propiamente no delibera, sino que trata de imponer una posición sus intereses individuales. 


\section{Conclusión}

Con lo dicho a lo largo del trabajo podemos concluir que la polarización de los procesos deliberativos en situaciones de desacuerdo es un problema que puede abordarse desde las mismas categorías de la democracia deliberativa. El modo de hacerlo será incidiendo precisamente en los aspectos que las aproximaciones hechas desde la neurociencia no son capaces de reconocer.

Efectivamente, como hemos señalado, la neurociencia concibe la deliberación como una interacción de procesos psicológicos: para Lakoff y Haidt esos procesos serían meras reacciones intuitivas; para Mercier y Landemore se trataría de mecanismos cognitivos desarrollados por la evolución. Pero ambos enfoques son claramente reduccionistas. Por un lado, porque olvidan la dimensión epistémica de la cognición humana. El razonamiento moral se rige por unos parámetros epistémicos como la orientación por un criterio de justicia, la pretensión de universalidad o la expectativa de convencimiento, que no son tenidos en cuenta desde la psicología evolutiva. Tampoco los marcos referenciales sobre los que se estructura la cognición humana pueden reducirse a meras estructuras mentales acuñadas por la evolución en nuestro cerebro. Porque nuestra aproximación a la realidad no se reduce a un mero cálculo inconsciente dirigido a la supervivencia individual o grupal, sino que parte del reconocimiento de unos bienes que trascienden nuestras inclinaciones o intereses. Finalmente, estos autores dejan de lado la dimensión moral de la deliberación y en particular de las emociones. Su aproximación reduccionista a la deliberación, y centrada en el estudio de procesos psicológicos, es ciega a la capacidad de las emociones morales para revelarnos cuándo los supuestos morales de la deliberación son violados por los participantes.

Sin estas dimensiones epistémica y moral, ausentes en el enfoque neuropsicológico, no puede ser analizada correctamente la deliberación y en consecuencia tampoco los procesos de polarización. Ciertamente, la polarización es un problema complejo que responde a diversos factores y que no se desarrolla de la misma forma en todos los contextos. Por ejemplo, existe una tendencia hacia la homogeneización de posiciones hacia la postura mayoritaria cuando se participa en procesos de debate gratuitos donde no se toma una decisión sobre el asunto tratado. En ellos la minoría no desea parecer descortés y tiende a acomodar su visión a la mayoritaria (Schkade, Sunstein y Hastie, 2010, 236). En cambio, en genuinos procesos deliberativos, dirigidos a la toma de decisiones sobre un asunto, el tratamiento de temas polémicos puede producir un enconamiento de posiciones antagónicas. En estos contextos de fuerte desacuerdo sobre el asunto a tratar, la polarización no responde a meras reacciones psicológicas inconscientes. Es en realidad fruto de las emociones reactivas de carácter moral surgidas ante el choque de marcos cognitivos en la deliberación y la futilidad de los argumentos empleados para cambiar la posición del interlocutor. Por ello, los consejos que desde la neuropsicología (o desde la ética) se aporten para reducir la polarización deberán conducirse hacia el cultivo de las emociones morales y a la aceptación de la limitada capacidad de acuerdo entre las personas debido a las diferencias cognitivas que separan a los sujetos. Afortunadamente algunos pasos se están dando en este sentido (Ferbach, Rogers, Fox y Sloman, 2013). 


\section{Bibliografía}

Auber, Johannes, Crott, Helmut y Werner, Johachim (1992): «Choice Shift and Group Polarization: An Analysis of the Status of Arguments and Social Decision Schemes», Journal of Personality and Social Psychology, vol. 62, pp. 50-61.

Aristóteles (2000): Retórica. Gredos, Madrid.

Benhabib, Seyla (1996): «Toward a deliberative model of democratic legitimacy», en: S. Benhabib (ed): Democracy and difference. Princeton University Press, Princeton.

Bohman, James (1996): Public deliberation. The MIT Press, Cambridge.

Castells, Manuel (2009): Comunicación y poder. Alianza, Madrid.

Christiano, Thomas (1996): The rule of the many. Westview Press, Colorado.

Cohen, Joshua (1986): «An epistemic conception of democracy», Ethics, vol. 97, pp. 26-38.

Cortina, Adela (2007): Ética de la razón cordial. Educar en la ciudadanía en el siglo XXI. Nobel, Oviedo.

Cortina, Adela (2011a): Neuroética y neuropolítica. Sugerencias para la educación moral. Tecnos, Madrid.

Cortina, Adela (2011b) «Ciudadanía democrática: ética, política y religión. XIX Conferencias Aranguren», Isegoría. Revista de Filosofía Moral y Política, núm. 44, pp. 13-55.

Darley, Jody L. y Rusbult, Caryl E. (2001): «Attitude alignment in close relationships», Journal of Personality and Social Psychology, vol. 81, pp. 65-84.

Ferbach, Philip M., Rogers, Todd, Fox, Craig R., Sloman, Steven (2013): «Political extremism is supported by an Illusion of understanding», Psychological Science, vol. 24,

Freeman, Samuel (2000): «Deliberative democracy. A Sympathetic comment», Philosophy and Public affairs, vol.29, núm. 4, 2000, pp. 371-418.

García-MarzÁ, Domingo (2012): «Neuropolítica: una mirada crítica sobre el neuropoder», en: Adela Cortina (ed.): Guía Comares de neurofilosofía práctica. Comares, Granada.

Gaus, Gerald F. (1997): «Reason, justification and consensus», en: J. Bohman y W. Rehg (eds): Deliberativedemocracy. Essays on reason and politics. The MIT Press, Cambridge, pp. 205-242.

Gutmann, Amy y Thompson, Dennis (1997): Democracy and disagreement. Belknap Press of Harvard University Press, Cambridge.

Habermas, Jürgen (1965): Conciencia moral y acción comunicativa. Crítica, Barcelona.

Habermas, Jürgen (1998): Facticidad y validez. Trotta, Madrid.

Haidt, Jonathan (2001): «The emotional dog and its rational tail. A social intuitionist approach to moral judgement», Psychological Review, vol. 108, pp. 814-834.

Haidt, Jonathan (2012): The righteous mind. Why good people are divided by politics and religion. Pantheon Books, New York.

Haidt, Jonathan y Bjorklund, Fredrik (2007): «Social Intuitionists answer six questions about moral psychology», en: W.Sinnott-Armstrong (ed.): Moral Psychology, vol. 2, The MIT Press, Massachusetts.

Isenberg, Daniel J. (1986): «Group polarization: A critical review and meta-analysis», Journal of Personality and Social Psychology, vol. 50, núm. 6, 1986, pp. 1141-1151.

Kunda, Ziva (1990): «The case for motivated reasoning», Psychological Bulletin, vol.108, pp. 480-498. 
Lakoff, George (2009): The political mind. A cognitive scientist guide to your brain and its politics. Penguin Books, New York.

Landemore, Hélène y Mercier, Hugo (2012): «Talking it out with others vs. deliberation within and the law of group polarization: Some implications of the argumentative theory of reasoning for deliberative democracy», Análise Social, vol. XLVII, núm. 205 (4º, pp. 910-934.

Lord, Charles G., Ross, Lee, y Lepper, Mark R. (1979): «Biased Assimilation and Attitude Polarization: The Effects of Prior Theories on Subsequently Considered Evidence», Journal of Personality and Social Psychology, vol. 37, pp. 2098-2109.

MacIntyre, Alasdair (1987): Tras la virtud. Crítica, Barcelona.

Manin, Bernard (2005): «Democratic deliberation: why we should promote debate rather than discussion». Paper presentado en el Program in Ethics and Public Affairs Seminar, Princeton University, octubre. Documento accesible en http://www.politics.as.nyu.edu/ docs/IO/2792/delib.pdf .

Markovits, Elizabeth (2006): «The trouble with be earnest: Deliberative Democracy and the sincerity norm», The Journal of Political Philosophy, vol. 14, núm. 3, pp. 249-269.

Mercier, Hugo (2011): "What good is moral reasoning?", Mind \& Society, vol. 10, núm. 2, pp. 131-148.

Mercier, Hugo (2012): «Some clarifications about the Argumentative Theory of Reasoning: A reply to Santibáñez Yáñez», Informal Logic, vol. 32, (2) 2012, pp. 259-268.

Mercier, Hugo y Landemore, Hélène (2012): «Reasoning is for arguing. Understanding the success and failures of deliberation», Political Psychology, vol. 33, núm.2, pp. 243-258.

Mercier, Hugo y Sperber, Dan (2011): «Why do humans reason? Arguments for an argumentative theory», Behavioral and Brain Sciences, vol. 34, pp. 57-111.

Paxton, Joseph M. y Greene, Joshua (2010): «Moral reasoning: hints and allegations», Topics in Cognitive Science, vol. 2 núm. 3, pp. 511-527.

Pérez Zafrilla, Pedro Jesús (2009): «Democracia Deliberativa: una apuesta por el valor epistémico de la deliberación publica», Revista de Filosofía, vol.34, pp. 155-73.

Pérez Zafrilla, Pedro Jesús (2016): «Is Deliberative Democracy an adaptive political theory? A critical analysis of Hugo Mercier's Argumentative Theory of Reasoning», Análise Social, vol. LI, núm. 220 (3), pp. 544-564.

Richardson, Henry (2002): Democratic autonomy. Oxford University Press, Oxford.

Schulz, Kathryn (2010): Being Wrong. Adventures in the margin of error. Portobello Books, New York.

Schkade, David, Sunstein, Cass R. y Hastie, Reid (2010): «When deliberation produces extremism», Critical Review, vol. 22, pp. 227-252.

Smith, Douglas G. (1997): «The illiberalism of liberalism: Religious discourse in the public square», San Diego Law Review, vol. 34, pp. 1.571-1.642.

Sperber, Dan, Clément, Fabrice, Heintz, Christopher, Mascaro, Oliver, Mercier, Hugo, Origgi, Gloria, y Wilson, Deirdre (2010): «Epistemic vigilance», Mind \& Language, vol. 25, pp. 353-393

Sunstein, Cass (2002): «The law of group polarization», Journal of Political Philosophy, vol. 10, pp. 175-195.

Talisse, Robert (2009): Democracy and moral conflict. Cambridge University Press, Cambridge. 
Taylor, Charles (1996): Las fuentes del yo. La construcción de la identidad moderna. Paidós, Barcelona.

Thompson, Dennis (2008): «Deliberative democratic theory and practical political science», Annual Review of Political Science, vol.11, pp. 497-520.

Westen, Drew (2007): The political brain. The role of emotion in deciding the fate of the nation. Public Affairs, New York.

Wojcieszak, Magdalena (2011): «Deliberation and attitude polarization», Journal of Communication, vol.61, pp. 596-617. 